\title{
Thermodynamics of the Ternary Water-Salt Systems
}

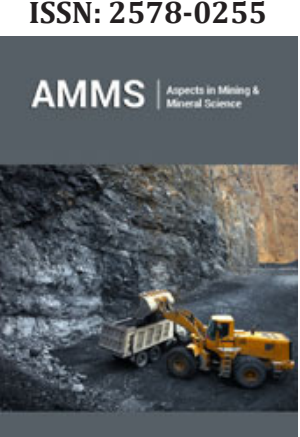

*Corresponding author: Allakhverdov GR, State Scientific-Research Institute of Chemical Reagents and High Purity Chemical Substances- National Research Centre, Kurchatov's Institute, Moscow, Russia

Submission:

Published: 笽 December 10, 2019

Volume 4 - Issue 3

How to cite this article: Allakhverdov GR ,Zhdanovich OA. Thermodynamics of the Ternary Water-Salt Systems. Aspects Min Miner Sci.4(3). AMMS.000586.2019. DOI: 10.31031/AMMS.2019.04.000586

Copyright@ Allakhverdov GR, This article is distributed under the terms of the Creative Commons Attribution 4.0 International License, which permits unrestricted use and redistribution provided that the original author and source are credited.

\section{Allakhverdov GR* and Zhdanovich $\mathrm{OA}$}

State Scientific-Research Institute of Chemical Reagents and High Purity Chemical SubstancesNational Research Centre, Kurchatov's Institute, Russia

\section{Abstract}

The article present methods for calculation the thermodynamics characteristics of ternary water-salt solutions based on data for binary solutions of the components that form a mixed solution.

Keywords: Osmotic coefficient; Isopiestic solution; Activity of component

\section{Introduction}

The calculation of the characteristics of mixed solution based on the properties of binary solutions is one of the central problems of the theory of solutions. The McKay-Perring equation [1] shows that the calculation of the activity coefficient of the components of mixed electrolyte solutions can be carried out by comparing the experimental data for mixed solutions with the data for binary solutions under the condition of isopiestic equilibrium of all solutions. This condition opens a window for other methods for calculation the properties of mixed solutions based on data tor binary solutions of the individual components of ternary system.

\section{Theory}

Suppose that two binary solutions of different electrolytes having the equal vapor pressure over a solution, when mixed, form a solution with the same vapor pressure. If in a mixed solution the concentration of one electrolyte $\mathrm{x}_{1}$ and another $\mathrm{x}_{2}$, then the following relation should hold $\frac{d x_{1}}{d x_{2}}=c$, where $\mathrm{C}=$ constant, since the solvent activity remain unchanged. Here $\mathrm{x}$ is the comparative concentration

$$
x=\frac{v m}{W}
$$

where $v$ is the stoichiometric coefficient of the electrolyte, $\mathrm{m}$ is molality, $\mathrm{W}$ is the number of moles solvent per $1 \mathrm{~kg}$ (for water $\mathrm{W}=55.51$ ). The molar fraction of the components of the mixed solution can be defined as

$$
f_{1}=\frac{x_{1}}{X} ; f_{2}=\frac{x_{2}}{X}
$$

where $x=x_{1}+x_{2}$, and further determine $\mathrm{C}$ as

$$
C=\frac{d x_{1}}{d x_{2}}=\frac{f_{1} \frac{d X}{d 1}+f_{1}+X}{\left(1-f_{1}\right) \frac{d X}{d f_{1}}-X}
$$

Separating variables and integrating Eq. (3), we obtain

$$
-\int_{X_{1}}^{X_{2}} \frac{d X}{X}=\int_{1}^{0} \frac{1+C}{f_{1}(1+C)-C} d f_{1}
$$

Hence, we have

$$
\frac{d x_{1}}{d x_{2}}=-\frac{X_{1}}{X_{2}}
$$

where $X_{1}$ and $X_{2}$ are the concentrations of isopiestic binary solutions. Integrating Eq. (5) taking into account the boundary conditions, we obtain [2]

$$
\frac{x_{1}}{X_{1}}+\frac{x_{2}}{X_{2}}=1(6)
$$


or, using Eq. (2)

$$
\frac{f_{1}}{X_{1}}+\frac{f_{2}}{X_{2}}=\frac{1}{X}
$$

Eq. (6), using Eq. (1), can be represented as

$$
\frac{m_{1}}{m_{1}^{*}}+\frac{m_{2}}{m_{2}^{*}}=1
$$

where $\mathrm{m}_{\mathrm{i}}$ is the molality of the components in mixed solution and $\mathrm{m}_{\mathrm{i}}^{*}$ is the molality of the components in binary isopiestic solutions. In this form Eq. (8) reflects the empirical rule by Zdanovsky [2], which shows a linear dependence of the inverse molality on the composition of the solution (Figure 1).

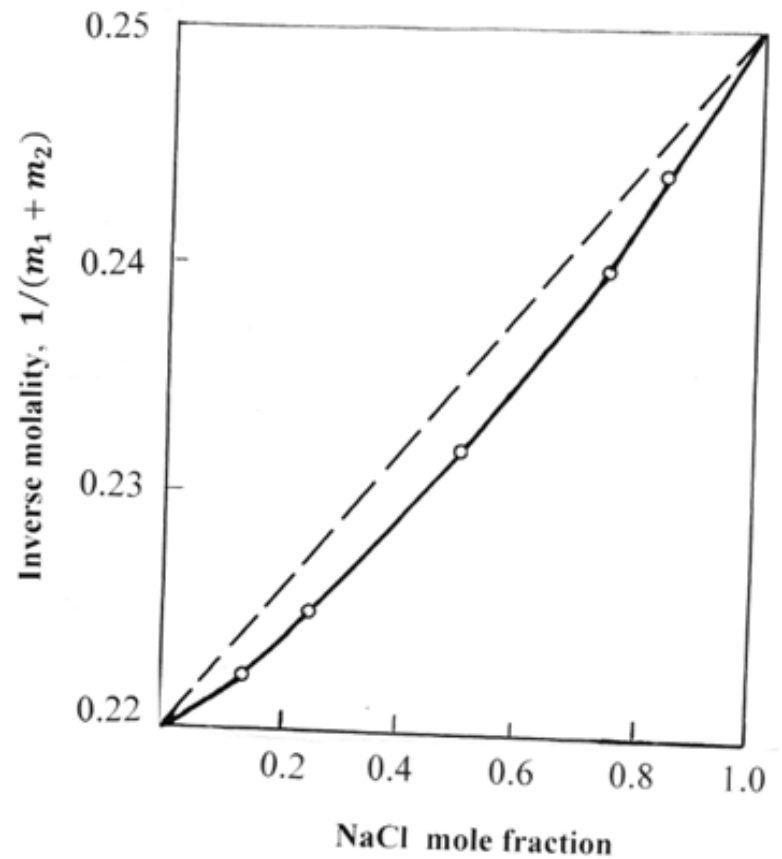

Figure 1: The composition of a mixed solution in the system NaCl-KCl-H2O at aw=0.851 ( $\mathrm{T}=298 \mathrm{~K})$. The dotted line according to Eq. (8).

At constant pressure and temperature, the Gibbs-Duhem equation for a ternary water-salt system can be represented as

$$
v_{1} m_{1} d \ln a_{1}+v_{2} m_{2} d \ln a_{2}+W d \ln a_{w}=0
$$

where $a_{w}$ is the solvent activity and $a_{i}$ is the activity of the component in the mixed solution, for example,

$$
a_{2}=\gamma_{2}\left(v_{2+} m_{2}\right)^{v_{2+} / v_{2}}\left(v_{1-} m_{1}+v_{2-} m_{2}\right)^{v_{2-} / v_{2}}
$$

where $v_{2}=v_{2+}+v_{2-}$ and $\gamma_{2}$ is activity coefficient in ternary solution. Substitution Eq. (10) into (9) and using Eq. (1), we have

$$
x_{1} d \ln a_{1}^{\prime}+x_{2} d \ln a_{2}^{\prime}+d \ln a_{w}=0
$$

where $a_{i}^{\prime}=x_{i} \gamma_{i}$. Combining Eq. (7) and (11) we obtain

$$
f_{1}\left(d \ln a_{1}^{\prime}+\frac{1}{X_{1}} d \ln a_{w}\right)+f_{2}\left(d \ln a_{2}^{\prime}+\frac{1}{X_{2}} d \ln a_{w}\right)=0
$$

On the other hand, for each binary solution it is possible to write

$$
d \ln a_{i}^{*}+\frac{1}{X_{i}} d \ln a_{w}=0
$$

where $a_{i}^{*}$ is the activity of the components in isopiestic binary solution. Combining Eq. (12) and (13) we obtain

$$
f_{1}\left(d \ln a_{1}^{\prime}-d \ln a_{1}^{*}\right)+f_{2}\left(d \ln a_{2}^{\prime}-d \ln a_{2}^{*}\right)=0
$$

The solution of Eq. (12) can be represented as

$$
a_{i}^{\prime}=f_{i} a_{i}^{*}
$$

Revealing the activity of the components in the binary isopiestic solutions: $a_{i}^{*}=X_{i} \gamma_{i}^{*}$, Eq. (15) can be transformed to the form

$$
\gamma_{i}=\gamma_{i}^{*} \frac{X_{i}}{X}
$$

or, moving on to molality

$$
\gamma_{i}=\gamma_{i}^{*} \frac{v_{i} m_{i}^{*}}{\sum v_{i} m_{i}}
$$

where $\gamma_{i}^{*}$ are activity coefficients in the binary isopiestic solution.

The McKay-Perring equation can be expressed in integral form as

$$
\ln \frac{a_{i}^{\prime}}{f_{i} a_{i}{ }^{*}}=\int_{1}^{a_{w}}\left[\frac{1}{X_{i}}-\frac{1}{X}-\left(1-f_{i}\right)\left(\frac{\partial \frac{1}{X}}{\partial f_{i}}\right)_{a_{w}}\right] d \ln a_{w}
$$

Substitution here Eq. (7) turns the integral expression into zero and Eq. (18) turns into Eq. (15). Thus, Eq. (15) is an approximate solution to Eq. (18). A more rigorous solution can be obtained using equation

$$
\frac{1}{X}=\frac{f_{1}}{X_{1}}+\frac{f_{2}}{X_{2}}+k f_{1} f_{2}
$$

where $\mathrm{k}$ is a constant coefficient. Then, according to [2], integrating Eq. (18) we have

$$
\ln \frac{\gamma_{i}}{\gamma_{i}^{*}}=\ln \frac{X_{i}}{X}+k\left(1-f_{i}\right)^{2} \ln \frac{X_{1} \gamma_{1}^{*}}{X_{2} \gamma_{2}}
$$

\section{Discussion}

Eq. (20) described very well the properties of ternary systems, but the coefficient $\mathrm{k}$ can only be determined experimentally. However, for most systems this coefficient is quite small, therefore, below we will consider the practical application of Eq. (7) and the associated Eq. (15-17), which allow us to calculate the activity of the components without involving experimental data for ternary systems. For two binary isopiestic solutions, the equality must be satisfied

$$
X_{1} \varphi\left(x_{1}\right)=X_{2} \varphi\left(x_{2}\right)
$$

where $\varphi$ is the osmotic coefficient. If form of function $\varphi(\mathrm{x})$ is known, then for a given concentration of one of the solutions, the concentration of other solution can be calculated. Function $\varphi(\mathrm{x})$, according to [3], can be represented as

$$
\varphi=1+\varphi_{e}+\varphi_{a}+\varphi_{h}
$$

where $\varphi_{\mathrm{e}^{\prime}} \varphi_{\mathrm{a}^{\prime}} \varphi_{\mathrm{h}}$ are bound up with electrostatic interaction, association and hydration ions, respectively. Further, using the known ratio

$$
\ln \gamma=\varphi-1+\int_{0}^{x}(\varphi-1) d \ln x
$$


the electrolyte activity coefficient can be defined as

$$
\gamma=\gamma_{e} \gamma_{a} \gamma_{h}
$$

where $\gamma_{\mathrm{e}^{\prime}} \gamma_{\mathrm{a}^{\prime}}, \gamma_{\mathrm{h}}$ are corresponding constituents:

$$
\begin{gathered}
\ln \gamma_{e}=-\frac{1}{6} L Q \sqrt{x} e^{-Q_{0} \sqrt{x}}-\frac{L}{3 r_{0}}\left(1-e^{-Q r_{0} \sqrt{x}}\right) \\
\ln \gamma_{a}=-\frac{2 \xi k_{a} x}{1+k_{a} x / 2}-4 \xi \ln \left(1+k_{a} x / 2\right) \\
\ln \gamma_{h}=\left(h x+2 h^{2} x^{2}\right)+\frac{1}{B}\left(h_{0}-h-h^{2} x\right)+\frac{1}{2 B^{2}}\left(h_{0}^{2}-h^{2}\right)
\end{gathered}
$$

where the first term of each equation is corresponding to $\varphi_{\mathrm{i}}$. Here $\mathrm{r}_{0}$ is ionic radius, $\mathrm{k}_{\mathrm{a}}$ is ion association constant, $h=h_{0} \exp (-B x)$ , where $\mathrm{h}_{0}$ is hydration number in infinitely dilute solution and $B$ is the decrement, $L=\left|z_{+} z_{-}\right| * 7.156 * 10^{-8} \mathrm{~cm}, Q=\left|z_{+} z_{-}\right|^{1 / 2} * 1.735 * 10^{8} \mathrm{~cm}^{-1}$, where $\mathrm{z}_{+}$and $\mathrm{z}_{+}$are the charges of ions, $\xi=v_{+} v_{-} / v^{2}$.

If the mixed solution is in equilibrium with the solid phase of one of the components, then in this scope the activity of the component of the solution remain constant and equal to its activity in a binary saturated solution. For example, in system $\mathrm{NaCl}-\mathrm{KCl}-\mathrm{H}_{2} \mathrm{O}$ (I-I valente electrolytes) we have

$$
a_{2}=\gamma_{2} m_{2}^{1 / 2}\left(m_{1}+m_{2}\right)^{\frac{1}{2}}=\gamma_{2}^{0} m_{2}^{0}
$$

where $\gamma_{2}{ }^{0}$ and $m_{2}{ }^{0}$ activity coefficient and molality, respectively, in saturated solution. On the other hand, comparing the mixed solution with binary isopiestic solutions of pure components and using Eq. (8), we obtain

$$
m_{1}=m_{1}^{*}-m_{2} \frac{m_{1}^{*}}{m_{2}^{*}}
$$

where $\mathrm{m}_{1}{ }^{*}$ is an arbitrarily chosen concentration. Substituting Eq. (29) and (17) into Eq. (28), we can determine the concentration of the component in the mixed solution $\mathrm{m}_{2}$ by the equation

$$
m_{2}\left[1-\left(1-\frac{m_{1}^{*}}{m_{2}^{*}}\right)\left(\frac{\gamma_{2}^{0} m_{2}^{0}}{\gamma_{2}^{*} m_{2}^{*}}\right)^{2}\right]=m_{1}^{*}\left(\frac{\gamma_{2}^{0} m_{2}^{0}}{\gamma_{2}^{*} m_{2}^{*}}\right)^{2}
$$

when $\mathrm{m}_{2}{ }^{*}$ can be defined, using Eq. (21) and functions $\varphi(\mathrm{x})$ for binary solutions of components. Further, using Eq. (24)-(27), we can define $\gamma_{2}{ }^{*}$. After that, we can determine $m_{1}$ according to Eq. (29). Similarly, calculation can be made when another component there is in the solid phase. Figure 2 shows the calculated and experimental data on the composition of saturated solutions in the ternary system. The component activity was calculated using Eq. (24-27); experimental data for osmotic coefficients in binary solutions were used as initial data to determine the parameters of equations [4]. These parameters were also using when the calculated concentrations were in the scope of oversaturated solutions, since the transition in this scope is not accompanied by a sudden change in the properties of the solution. Considering the ternary water-salt systems in another aspect, it is possible to evaluate the efficiency of the separation of inorganic substances during crystallization from solutions. For inorganic compounds that do not form solid solution, according Ref. [5], the co-crystallization coefficient can be defined as

$$
D_{2}=\frac{y_{2} / y_{1}}{x_{2} / x_{1}}=\frac{a_{1}^{0}}{a_{2}^{0}} \frac{a_{2}^{*}}{a_{1}^{*}}
$$

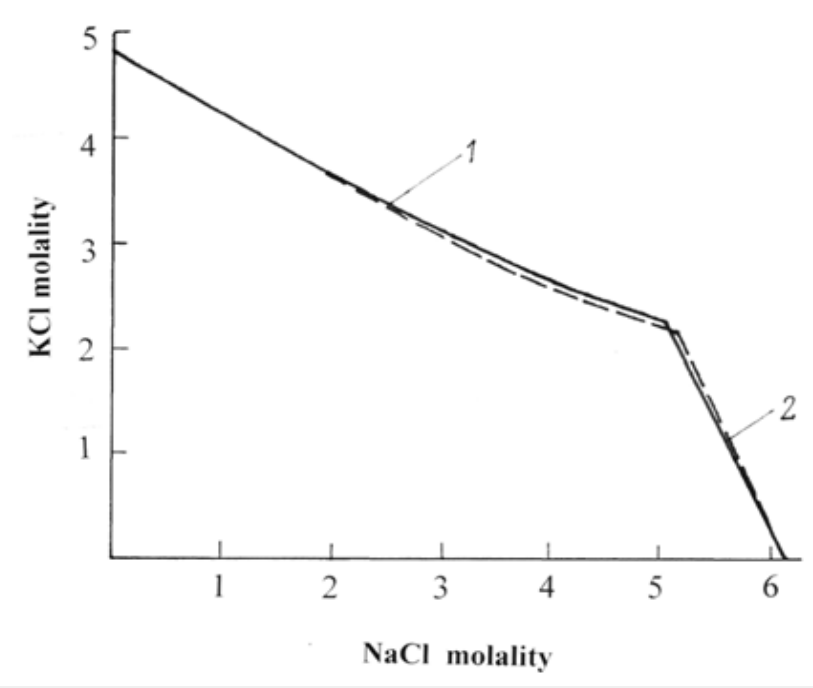

Figure 2: Solubility in the systems $\mathrm{NaCl}-\mathrm{KCl}-\mathrm{H} 2 \mathrm{O}$ at $298 \mathrm{~K}$.

1-saturated solutions of potassium chloride.

2-saturated solutions of sodium chloride.

The solid line is experimental data [4].

The dotted line is calculated data.

where $y_{i}$ is the molar fraction of the component in solid phase. In the limiting case when $\mathrm{x}_{2} \rightarrow \rightarrow 0$ also $\mathrm{a}_{1}{ }^{*} \cdots \rightarrow \mathrm{a}_{1}{ }^{0}$ and co-crystallization coefficient of impurity component can be expressed as

$$
D_{2}^{0}=\lim _{x_{2} \rightarrow 0} \frac{x_{1} y_{2}}{y_{1} x_{2}}=\frac{a_{2}^{*}}{a_{2}^{0}}
$$

Table 1: Cocrystallization coefficients in ternary systems at $298 \mathrm{~K}$.

\begin{tabular}{|c|c|c|}
\hline System & $\mathbf{D}_{2}{ }^{\circ}$ (calc.) & $\mathbf{D}_{2}{ }^{\circ}$ (meas.) \\
\hline $\mathrm{KNO}_{3}-\mathrm{KCl}-\mathrm{H}_{2} \mathrm{O}$ & 0.444 & 0.454 \\
\hline $\mathrm{KNO}_{3}-\mathrm{Mg}\left(\mathrm{NO}_{3}\right)_{2}-\mathrm{H}_{2} \mathrm{O}$ & 0.032 & 0.035 \\
\hline $\mathrm{KNO}_{3}-\mathrm{Co}\left(\mathrm{NO}_{3}\right)_{2}-\mathrm{H}_{2} \mathrm{O}$ & 0.028 & 0.030 \\
\hline $\mathrm{KNO}_{3}-\mathrm{Cu}\left(\mathrm{NO}_{3}\right)_{2}-\mathrm{H}_{2} \mathrm{O}$ & 0.016 & 0.018 \\
\hline $\mathrm{KNO}_{3}-\mathrm{Al}\left(\mathrm{NO}_{3}\right)_{3}-\mathrm{H}_{2} \mathrm{O}$ & 0.035 & 0.036 \\
\hline
\end{tabular}




\section{References}

where $a_{2}^{*}=m_{2}^{*} \gamma_{2}^{*}$ is the activity of impurity component in its binary solution, which isopiestic to the saturated of the main component. Using Eq. (21) and functions $\varphi(x)$ for binary solutions of components, we can define $\mathrm{m}_{2}{ }^{*}$ and further $\gamma_{2}{ }^{*}$, using Eq. (24). The calculated and experimental data [6] of co-crystallization coefficient for some systems, where the main component is potassium nitrate, are given in Table 1.

\section{Conclusion}

Thus, using the example of solubility and co-crystallization of components in ternary water-salt systems, the possibility of calculating the properties of ternary systems using properties of binary solution of individual components has been shown, which is of theoretical and practical interest for the technology of processing mineral raw materials.

1. McKay HAC, Perring JK (1953) Calculations of the activity coefficients of mixed aqueous electrolytes from vapor pressures. Trans Faraday Soc 49(363): 163-165.

2. Allakhverdov GR, Stepin BD (1973) Some appropriateness in thermodynamics of mixed electrolyte solutions. Zh Phys Chem (Rus) 47(2): 386-389.

3. Allakhverdov GR (2019) Thermodynamics of the electrolyte solutions. AMMS 4(1): 465-474.

4. Mikulin GI (1968) Current issues in the physical chemistry of electrolyte solutions. Leningrad, Russia.

5. Allakhverdov GR, Zhdanovich OA (2019) Theoretical aspects of obtaining pure inorganic substances during crystallization from solutions. AMMS 3(2): 396-397.

6. Allakhverdov GR, Mikhlin AL (2017) Separation factors for the crystallization of inorganic substances from aqueous solutions. Inorganic materials 53(5): 525-528.

For possible submissions Click below: 